\title{
Experimental and theoretical bases for mechanisms of antigen discrimination by $T$ cells
}

\author{
Masashi K. Kajita ${ }^{1}$, Ryo Yokota ${ }^{2}$, Kazuyuki Aihara, ${ }^{1,2}$ and Tetsuya J. Kobayashi ${ }^{2}$ \\ ${ }^{1}$ Department of Mathematical Informatics, Graduate School of Information Science and Technology, The University of Tokyo, 4-6-1 \\ Komaba Meguro-ku, Tokyo 153-8505, Japan \\ ${ }^{2}$ Institute of Industrial Science, The University of Tokyo, 4-6-1 Komaba Meguro-ku, Tokyo 153-8505, Japan
}

Received October 2, 2014; accepted February 1, 2015

Interaction only within specific molecules is a requisite for accurate operations of a biochemical reaction in a cell where bulk of background molecules exist. While structural specificity is a well-established mechanism for specific interaction, biophysical and biochemical experiments indicate that the mechanism is not sufficient for accounting for the antigen discrimination by $T$ cells. In addition, the antigen discrimination by $T$ cells also accompanies three intriguing properties other than the specificity: sensitivity, speed, and concentration compensation. In this work, we review experimental and theoretical works on the antigen discrimination by focusing on these four properties and show future directions towards understanding of the fundamental principle for molecular discrimination.

Key words: specificity, sensitivity, speed, concentration compensation, kinetic proofreading

Cells use chemical reactions in various ways such as signal transduction, DNA transcription, and DNA replication. In most of these processes, the biochemical molecules engaging in the reactions must react with specific target molecules in order to realize accurate processing. However, various molecular species exist inside and outside of cells as background. In this circumstance, interaction with the specific targets is not necessarily guaranteed without discriminating

Corresponding author: Masashi K. Kajita, Department of Mathematical Informatics, Graduate School of Information Science and Technology, The University of Tokyo, 4-6-1 Komaba Meguro-ku, Tokyo 153-8505, Japan.

e-mail: mkajita@sat.t.u-tokyo.ac.jp the targets out of the other backgrounds. A famous mechanism of this molecular discrimination process is the substrate specificity in enzyme-catalyzed reactions. In general, the mechanism of the substrate specificity is structural specificity such as induced fit or conformational selection [1-4]. While the structural specificity is a well-established mechanism, it cannot explain all the molecular discrimination processes. The antigen discrimination by $\mathrm{T}$ cells is such an example where reaction networks may be relevant for realizing specificity. Recent biophysical experiments are now providing new insights on the mechanisms, and intriguing ideas have also been proposed theoretically.

\section{Characteristics of T Cell Antigen Discrimination}

$\mathrm{T}$ cells are a type of the immune cells that play a crucial role in the adaptive immunity to detect and exclude the invasions of pathogens and the emergence of cancer cells. T cells detect their targets via peptide-antigens presented on the surface of the antigen presenting cells (APCs) using the T cell receptor (TCR) [5]. T cells can recognize various different peptides to protect the whole body from different pathogens because each $\mathrm{T}$ cell has TCRs with different structure from others with high probability. This diversity is generated by the random genetic recombination of the TCR genes [6].

One outstanding property of the $\mathrm{T}$ cells other than their diversity is that it can detect a small amount of its target antigens sensitively and specifically out of similar non-target ones. In addition, the T cells can sense the arrival of the target antigens quickly by effectively compensating the influence from the abundant non-target antigens in background. Therefore, the antigen discrimination by $\mathrm{T}$ cells can be characterized by the four important properties: namely, specific- 
ity, sensitivity, concentration compensation, and speed. The mechanism of the antigen discrimination and its relation to the four properties is one of the fundamental issues in immunology and yet to be clarified. About the mechanism and the properties, new insights have been delivered recently by the elucidation of its detailed molecular basis and by the quantitative measurements of single-cell dynamics [7]. Such molecular and quantitative information now serves as the firm support for theoretical investigation on the fundamental mechanisms of the antigen discrimination. In this paper, we review experimental and theoretical works on the antigen discrimination by focusing on the four properties and show future directions on this issue.

\section{Specificity}

The most important characteristic of the antigen discrimination by $\mathrm{T}$ cells is the ability to discriminate the targetantigens specifically. A $T$ cell recognizes antigens using the TCRs on the membrane of the $\mathrm{T}$ cell. $\mathrm{B}$ cells also recognize antigens using B cell receptors (BCRs) or antibodies. In contrast to the BCR that interacts with an antigen directly, the TCR cannot bind to an antigen directly [8]. A TCR can only recognize antigen-peptide on major histocompatibility complex (MHC). An antigen peptide is a short peptide fragment with about 10 amino acids held in the groove of an MHC molecule [8]. Compared with antibodies, the affinity of a TCR to a pMHC is relatively low, and both target and nontarget antigens have similar low affinities to the TCR [9].

In addition to the low affinity of the TCR to antigens, the short sequence of the antigen peptides with the limited diversity results in the small difference between the target and non-target antigen peptides. Despite the low affinity and the small difference in the sequence variety, the T cells can discriminate the target antigens specifically.

\section{Antigen similarity based on peptide-sequence}

The specificity of the T cells has been tested by changing the antigen sequences. Several studies have shown that $\mathrm{T}$ cells can discriminate a target antigen from an analog peptide whose sequence is replaced only by a single amino acid from the target one $[10,11]$. Monks et al. also verified that the analog peptide cannot induce the formation of immunological synapse, a cluster of TCRs and other receptors [12].

The crystal structure analysis clarified that TCR-pMHC complexes of the target and non-target antigens have almost no conformational difference without the interaction between co-receptors on the surface of the APC and the T cell $[13,14]$. This result indicates that the mechanism of the antigen discrimination by $\mathrm{T}$ cells is different from the structural specificity in a G protein-coupled receptor (GPCR) and Calmodulin, which accompanies large conformational change in the discrimination process $[4,14,15]$.

\section{Affinity based discrimination}

If the conformational change is not relevant, how can a $\mathrm{T}$ cell discriminate antigens? The formation of a TCR-pMHC complex initiates the binding of co-receptors to the complex, and this binding, in turn, phosphorylates the TCR [16]. Because such cascading reactions generally depend on the stability of the initiation complex, the strength of the affinity between the TCR and the pMHC may be related to the antigen discrimination.

From this idea, affinity-related parameters have been investigated quantitatively to understand what kinds of parameters are more relevant for the discrimination of antigens.

The affinity between a TCR and a pMHC is characterized by the binding rate $k_{\text {on }}$, the unbinding rate $k_{\text {off }}$ or the dissociation rate constants $K_{\mathrm{D}}=k_{\mathrm{off}} / k_{\text {on }}$ in the equilibrium condition, as follows:

$$
\mathrm{TCR}+\mathrm{pMHC} \underset{k_{\mathrm{off}}}{\stackrel{k_{\mathrm{on}}}{\rightleftarrows}} \mathrm{TCR} \cdot \mathrm{pMHC} .
$$

These parameters have been measured by using Surface Plasmon Resonance (SPR) [9,17-20]. While the SPR can measure the average of the affinity parameters in a $3 \mathrm{D}$ bulk solution, a TCR and a pMHC interact, in reality, on a 2D contact surface between a T cell and an APC. This problem is recently resolved by combining the fluorescence resonance energy transfer (FRET) and the total internal reflection fluorescence microscopy (TIRF) to measure the 2D parameter values for each single molecule [21]. While the SPR and the FRET with TIRF provide different parameter values, both measurements suggest that a non-target antigen forms a less stable complex with a TCR (i.e., larger $K_{\mathrm{D}}$ ) than a target-antigen $[9,20,21]$. Other groups also support the correlation between T-cell activation and the affinity of the antigens characterized by $K_{\mathrm{D}}$ and $k_{\text {off }}[22-25]$.

\section{Amplification of small difference by reaction network}

All the results described so far demonstrate that the difference of the affinity between the target and the non-target antigens is small and analog, whereas the response of the $\mathrm{T}$ cell to these antigens is discrete and digital. This observation implies that the small difference in the affinity is specifically amplified in all-or-non manner by the reaction networks downstream to the TCR-pMHC binding.

Kinetic proofreading is a mechanism that can amplify the small difference in the affinity. This mechanism was proposed by Hopfield and Ninio independently such as specific discrimination of transfer RNAs by a ribosome [26,27], and then McKeithan applied their idea to explain the specificity of the T cells to the antigens [28] (Fig. 1).

In the original model by Hopfield, he firstly considered a simple Michaelis-Menten-type enzymatic reaction as a model of the translation where the ribosome and the tRNAs play roles as enzyme and substrates, respectively. In such a reaction, the speed of the product formation for different substrates is linearly dependent on the ratio of their dissociation 
A

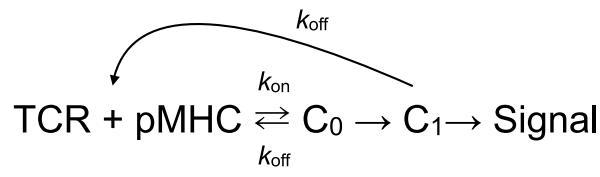

B

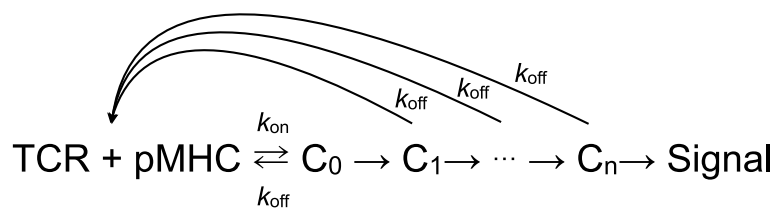

Figure 1 Schematic diagrams of the kinetic proofreading models. (A) The TCR-pMHC complex has several states $\left(\mathrm{C}_{0} \sim \mathrm{C}_{1}\right)$, and the fully activated state $\left(\mathrm{C}_{1}\right)$ can generate output signal. The rate constants, $k_{\text {on }}$ and $k_{\text {off }}$, represent the binding and the unbinding rates of the TCR pMHC interaction, respectively. A non-target antigen with low affinity only produces a partially activated complex. (B) Multiple states of the complex $\left(\mathrm{C}_{0} \sim \mathrm{C}_{\mathrm{n}}\right)$ can increase specificity of the antigen discrimination $[26,28]$.

rates in the equilibrium state. Therefore, the small difference in the affinities of substrates cannot be amplified. What he has shown was that this linear dependence on the affinity can be nonlinear by introducing two additional reaction pathways. One pathway irreversibly converts the substrateenzyme complex into an intermediate state as in Figure 1. The other is a dissociation reaction of the intermediate complex back to the dissociated enzyme and substrate (Fig. 1). These two pathways make the reaction non-equilibrium, and nonlinearly amplify the small difference in the affinity at a cost of energy consumption. He also demonstrated that multiplication of the pathways increases the nonlinearity, and thereby the small difference is exponentially amplified.

McKeithan casted this mechanism to the antigen discrimination of $\mathrm{T}$ cells by identifying the enzyme and the substrates in the kinetic proofreading with the TCR and the antigens [28]. He suggested that the multiple intermediate states in the proofreading can be interpreted as multiple phosphorylation states in the TCR or other modifications. As with the original model of the kinetic proofreading, the small difference in affinity between the target and the non-target antigens is exponentially amplified as the number of the irreversible intermediate states increases.

This theoretical hypothesis was experimentally validated by Kersh et al. [29]. They showed that target antigens sufficiently phosphorylate a TCR but non-target antigens partially phosphorylate the TCR. The results support the kinetic proofreading as the mechanism of the antigen discrimination by $\mathrm{T}$ cells.

However, the kinetic proofreading has several limitations. The multiple intermediate reactions reduce the probability and elongate the time for the TCR to reach the final state. Thereby, the proofreading impairs both the sensitivity to a small amount of the target antigens and the speed of the antigen discrimination. Therefore, an alternative mechanism that can resolve the incompatibility of the specificity, the sensitivity and the speed is necessary for understanding the antigen discrimination [30-33].

\section{Sensitivity}

In addition to the specificity, sensitivity is also an important characteristic of the T-cell-antigen-discrimination. Most of antigens presented by an APC to a T cell are non-target antigens including self-antigens. In addition, the number of antigens that a $\mathrm{T}$ cell can recognize is few. Therefore, $\mathrm{T}$ cells have to detect very few target antigens on the surface of the APCs. Considering this situation, sensitivity is requisite for the antigen discrimination by $\mathrm{T}$ cells.

\section{Single-cell-level activity}

A single cell imaging demonstrated that less than ten target antigens can actually activate $\mathrm{T}$ cells [34], and recent single molecule imaging further revealed that a single target antigen can activate a $T$ cell [35]. As a mechanism for the sensitivity, Stefanová et al. also experimentally showed that the activation and the sensitivity of $\mathrm{T}$ cells are regulated by a positive feedback of an extracellular signal-regulated kinase (ERK) and a negative feedback of the Src homology 2 domain phosphatase-1 (SHP-1) [36]. Even if the target antigens are few, the signal is amplified enough to activate a $\mathrm{T}$ cell by the positive feedback that enhances the output of the kinetic proofreading for the target antigens with high affinity. From these results, they proposed that this feedback regulation is relevant for sensitivity and specificity in the T cell antigen discrimination.

By following the work by Stefanová et al., Altan-Bonnet's group investigated the feedback regulation mechanism by a mathematical model including multiple states of TCRs as in the case with the kinetic proofreading [37,38] (Fig. 2). In this model, they assumed that a partially phosphorylated TCR activates SHP-1 that negatively regulates the TCR acti-

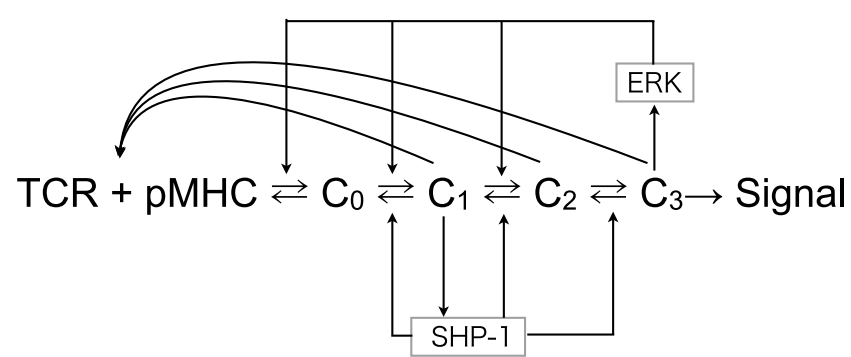

Figure 2 A diagram of the feedback model by Altan-Bonnet's group. Partially phosphorylated TCR (e.g. $\mathrm{C}_{1}$ ) activates SHP-1 that negatively regulates TCR activation by dephosphorylation. ERK is activated by the fully-phosphorylated TCR and positively regulates the TCR activation by protecting the interaction of TCR with SHP-1. When the antigen is non-target, the negative feedback dominates the positive one, leading to no activation of T cells. When the antigen is a target, in contrast, the positive feedback dominates the negative one to induce the full activation of T cells [37]. 
A

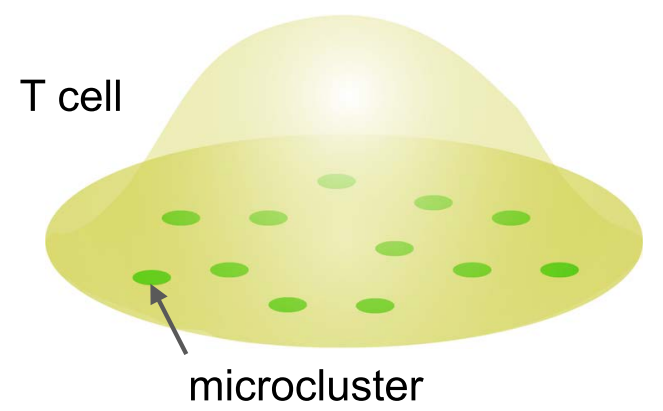

B

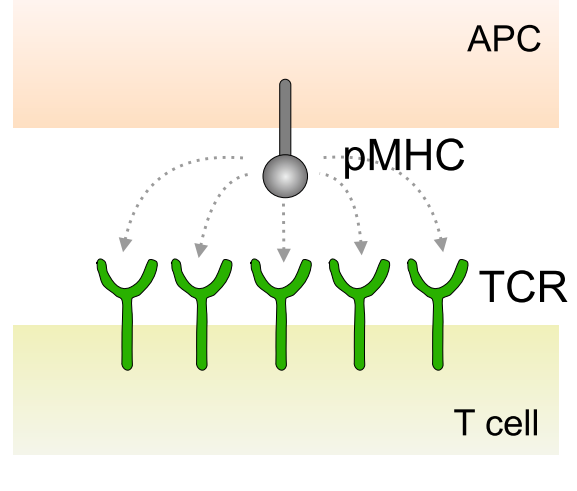

Figure 3 A concept of sensitivity enhancement by the TCR clustering and the serial triggering. (A) TCR microclusters are formed when antigens are presented to a T cell. (B) Serial TCR triggering in a microcluster by few antigens. When the TCR-pMHC complex has low affinity, a pMHC can interact with multiple TCRs because of the short lifetime of TCR-pMHC complex, and it will help a T cell to sense few antigens.

vation by dephosphorylation. In addition, ERK was assumed to be activated by fully-phosphorylated TCRs and positively regulate the TCR activation by protecting the interaction of the TCRs with SHP-1. When the antigen is non-target, the negative feedback dominates the positive one, leading to no activation of $\mathrm{T}$ cells. When an antigen is the target, in contrast, the positive feedback dominates the negative one to induce the full activation of $\mathrm{T}$ cells. The combination of the positive and the negative feedbacks, therefore, produces all or none response and balances the specificity and sensitivity. These results were experimentally confirmed by AltanBonnet's group in $[37,38]$.

\section{Microcluster Level Activity}

As other mechanisms for the sensitivity, TCR clustering and fast rebinding with low affinity of TCR-pMHC complex were recently proposed $[35,39,40]$. Just after the contact of a T cell with an APC, the formation of microclusters which are clusters of TCRs and other molecules is induced on the cell surface [41-43]. Manz et al. reported that the minimal triggering unit for the $\mathrm{T}$ cell activation (calcium signaling) is probably at least four pMHCs in a single cluster [44]. Huang et al. also showed that a single TCR microcluster contains about 100 TCRs within $2 \mu \mathrm{m}^{2}$ around a single pMHC, 1 minute after the antigen presentation [35]. These results suggest that a single pMHC recruits multiple TCRs to engage in the microcluster formation [35]. The low affinity of TCR-pMHC complex discussed in the previous section may be relevant for recruiting a large number of TCRs by only a single pMHC $[35,45,46]$.

In addition, clustering of TCRs around a few antigens might boot the sensitivity to the low concentration of the target antigens by enhancing the rebinding of the antigens. The antigens with high unbinding rates have been thought to be unable to activate T cells. However, Govern et al. showed that such antigens can activate $\mathrm{T}$ cells when they have high binding rates [47]. Because of the high binding and unbinding rates, the antigens can rebind to the same TCR several times which, in turn, leads to the sufficient total binding time for $\mathrm{T}$ cell activation. Valitutti et al. also reported that a small number of peptide-MHC complexes can serially engage and trigger up to 200 TCRs [45]. Taken together, these findings suggest that, when TCRs form a cluster, a few antigens can activate TCRs in the cluster one after another, which helps to increase sensitivity (Fig. 3).

\section{Concentration Compensation}

When an APC displays antigens to a T cell, a large number of non-target antigens are presented together with few target antigens. Therefore the $\mathrm{T}$ cell compensates the influence from the non-target antigens by preserving the sensitivity to the few target antigens.

François et al. proposed that the feedback mechanism is related to the concentration compensation based on a numerical simulation and theoretical analysis using the simplified model proposed by Altan-Bonnet's groups $[37,38,48]$. As we mentioned before, the original model by Altan-Bonnet's group is a combination of the kinetic proofreading, the ERK-dependent positive feedback, and the SHP-1-dependent negative feedback. François et al. simplified their models by removing the ERK-dependent positive feedback. The modified feedback model was shown to have weak dependency on the antigen concentration and a large number of non-target antigens are compensated. In addition, Lipniacki et al. reported that another feedback model focusing on the stochastic effect and bistability also shows compensation for a large number of non-target antigens [49]. However, further investigates are required on this problem.

\section{Speed}

To reduce influence by the infection, the immune system has to detect pathogens as quickly as possible when they invade a body. Because $\mathrm{T}$ cells initiate various immune responses by other lymphocytes, the speed of the antigen 
discrimination is crucial.

T cells contact with APCs in lymph nodes and discriminate pMHCs presented by the APCs. Recently, the cell-tocell interactions in vivo were observed by using the multi-photon microscopy. The imaging also revealed that a T cell is activated within 1-5 minutes after contact to an APC with the target antigen [50]. The in vivo imaging revealed that the contact of a T cell with an APC lasted about 8 minutes with antigens whereas the contact duration is shortened to 3.8 minutes without antigens.

$\mathrm{Ca}^{2+}$ flux is first induced in a $\mathrm{T}$ cell about one minute after the antigen presentation by an APC [34]. Concurrently, the formation of the microclusters starts [51], and the expansion of the T cell was also observed [51]. After about two minutes, the microclusters move to the center of the T-cell-APC contacting area and eventually merge into a supramolecular activation cluster (SMAC) or immunological synapse in 5 minutes. After these events, the T cell finally generates cytokines such as TNF- $\alpha$ or IL in about 40 minutes [35]. These results suggest that $\mathrm{T}$ cells are able to discriminate antigens in 1-5 minutes after the antigen presentation.

As a mathematical model to balance the specificity and speed, Murugan et al. proposed a general molecular discrimination model that was inspired by microtubule dynamics [52]. Microtubules, a component of the cytoskeleton, show dynamical switching between growing (rescue) and shrinking (catastrophe) phases called dynamic instability. The average length of the microtubule filament is determined by competition of the rescue and the catastrophe. When the rescue dominates the catastrophe, a simple model of microtubles can show unbounded growth of length whereas the length will be bounded on average when the catastrophe is dominant.

Motivated by this transition phenomenon, Murugan et al. generalized the kinetic proofreading model by proposing a ladder network in which the length of a microtuble is identified with the multiple states in the kinetic proofreading model. As is in the case with the microtubles, they also introduced the switching dynamics between two phases where the substrate moves between the multiple states in the for-

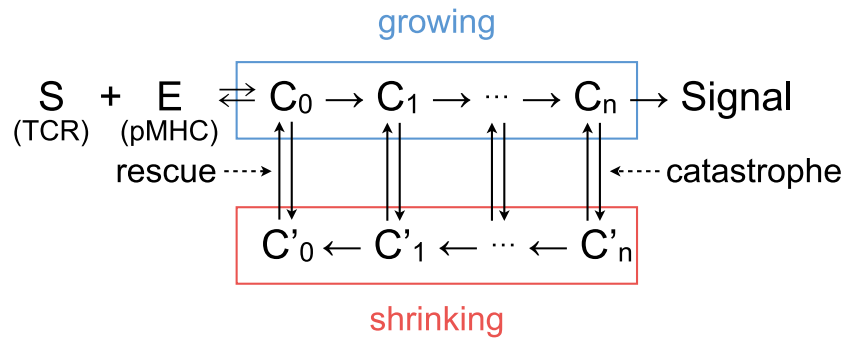

Figure 4 A modified ladder network model for kinetic proofreading. The complex of substrates $\mathrm{S}$ (i.e., TCR) and enzyme $\mathrm{E}$ (i.e., $\mathrm{pMHC})$ are switching between two phases $\left(\mathrm{C}_{\mathrm{s}}\right.$ and $\left.\mathrm{C}_{\mathrm{s}}^{\prime}\right)$. When the substrate is in the growth phase $\left(\mathrm{C}_{\mathrm{s}}\right)$, a reaction can proceed only in the forward direction. When the substrate is in the shrinking phase $\left(\mathrm{C}_{\mathrm{s}}^{\prime}\right)$, only a backward reaction occurs [52]. ward and backward directions, respectively as in Figure 4. The type of enzyme (antigens) is assumed to influence the frequency of the switching between the forward and backward modes. Depending on antigens, therefore, this model can show the transition between unbounded and bounded growth of substrate modification. They demonstrated that fast and specific discrimination can be achieved when the target and non-target antigens induce unbounded and bounded growth, respectively even though their difference is small. Because their model considers single molecule dynamics of the substrate, it can also accompany sensitivity. While this model is fascinating, its relation with the TCR clustering is still unclear, and needs further investigation.

\section{Summary and Discussion}

Here, we discuss possible feature directions of the research on the antigen discrimination by comparing its history with that of biophysics of gradient sensing in chemotaxis. Both the antigen discrimination and the gradient sensing can be regarded as kinds of cellular information processing in which the information processed is types of molecular species and molecular concentration, respectively. Compared with the chemotaxis, quantitative biophysical research of the antigen discrimination (more generally, molecule discrimination) is still lag behind in various aspects [53]. Therefore, the knowledge from the chemotaxis research is useful for foreseeing the upcoming challenges in the research of the molecule discrimination.

In the chemotaxis research, the key molecular components were identified by molecular biological techniques, and quantitative experiments have been established from singlecell-level chemotaxis tracking down to single-molecule imaging of the molecular components. Supported by these experimental data on input-output relations of the system, the accuracy of chemotaxic information processing (the gradient sensing) was tested both theoretically and experimentally, and their consistency was verified [54]. The molecular origin of sensing noise of gradient and its amplification mechanisms in signaling networks were also investigated by taking the quantitative physiological parameters into account [55]. Furthermore, the theoretical limits of the accuracy of the gradient sensing and the implementation of optimal sensing by chemical reactions were derived by employing the knowledge of statistics and information theory [56]. Such analysis also revealed that spontaneous cellular response in the chemotaxic networks may be linked to statistically optimal gradient sensing, which has been qualitatively suggested in biophysics [57]. Furthermore, a thermodynamic cost of sensing is recently attracting attention [58].

By taking the situation of chemotaxis into consideration, we think that the following approach will be important and necessary for further understanding of the molecular discrimination. Molecular immunology has already revealed many signaling molecules that are relevant for $\mathrm{T}$ cell antigen 
discrimination. The accuracy of discrimination has also been investigated quantitatively by focusing the affinity between antigens and receptors. Nonetheless, the proposed mechanisms to nonlinearly amplify the information of molecular difference are only the kinetic proofreading while several different mechanisms to amplify concentration information were found. It is crucial to obtain the list of fundamentally different mechanisms of the molecular discrimination in order to investigate quantitative experimental data from various perspectives. In addition, most of quantitative measurements on the antigen discrimination have been based on biochemical experiments in which a bulk of cells was used. It is only recently that the dynamics of molecular components for discrimination is observed in the single-cell-level by the time-lapse fluorescent imaging. This is one major difference from the chemotaxis research where output of the gradient sensing can be obtained by monitoring movement of single cells with bright field microscopes. In order to exploit the new dynamic information, similar mathematical techniques used in the chemotaxis can be applied to molecule discrimination. Among others, information-theoretical approach is not yet employed in order to analyze a physical limit of the molecule discrimination. As demonstrated in the chemotaxis research, such theory can be used to predict molecular networks that can achieve such physical limit $[56,57]$. This kind of information may be essential to narrow down the list of molecular components so that we can focus on the components that play the fundamental roles in discrimination.

In contrast, the relation between the efficiency of information processing and the non-equilibrium reaction has been pointed out since the early works of the molecule discrimination $[26,52]$ because the kinetic proofreading reaction fundamentally requires irreversible reactions that consume chemical energy from ATP, GTP and so on. However, the experimental verification is far behind these theoretical predictions. It is very important to fill this gap between theory and experiment. In addition, the theory of non-equilibrium thermodynamics and its relation to information processing is still developing. Recently, the field called information thermodynamics has clarified the trade-off between the energy consumption and the information gain, and it is now applied to cellular information processing systems $[59,60]$. These studies may help to evaluate the physical limit of molecular discrimination more appropriately and to propose good experimental setup for its verification.

In order to understand an unknown system, theoretically reproducing the behavior is indispensable. To this end, the detailed mathematical models developed for the antigen discrimination can actually contribute. However, reproduction itself is not sufficient to understand what is going on in the system. The theoretical approaches listed here (e.g., information theory and statistical physics) enable us to reveal functional difference of molecular systems whose properties cannot be well understood just by reproduction. The roles of clustering of TCR and other molecules clustering might be a good example. We can imagine multiple mechanisms that generate clusters of molecules. However, if this clustering is related to the molecule discrimination, the actual mechanisms of the clustering must be better than others with respect to the efficiency of discrimination. The comparison between actual accuracy and theoretical limits might clarify novel mechanisms of efficient cellular information processing, which is far beyond the current kinetic view of understanding of the molecule discrimination. We believe, therefore, that the extension of information theory and information thermodynamics is an impending challenge for further understanding of the molecular discrimination.

Finally we note that both molecular species and concentrations are simultaneously processed in most of intracellular systems in general. The chemotaxis and the antigen discrimination are just instances where one aspect of more general information processing appears substantially. When we reach sufficient understanding of both phenomena, we may be able to embark for a more long-term challenge to integrate them together.

\section{Acknowledgment}

This work was supported by Grant-in-Aid for JSPS Fellows, JSPS KAKENHI Grant Number 269545 and also supported by Platform for Dynamic Approaches to Living System from the Ministry of Education, Culture, Sports, Science and Technology, Japan.

\section{References}

[1] Fischer, E. Einfluss der configuration auf die wirkung der enzyme. Berichte der Dtsch. Chem. Gesellschaft 27, 29852993 (1894).

[2] Koshland, D. E. Application of a theory of enzyme specificity to protein synthesis. Proc. Natl. Acad. Sci. USA 44, 98-104 (1958).

[3] Koshland, D. E. The key-lock theory and the induced fit theory. Angew. Chemie Int. Ed. English 33, 2375-2378 (1995).

[4] Changeux, J. P. \& Edelstein, S. Conformational selection or induced fit? 50 years of debate resolved. F1000 Biol. Rep. 3, 19 (2011).

[5] Huppa, J. B. \& Davis, M. M. T-cell-antigen recognition and the immunological synapse. Nat. Rev. Immunol. 3, 973-983 (2003).

[6] Parham, P. The immune system, Fourth Edition (Garland Science, New York, 2014).

[7] Huang, J., Meyer, C. \& Zhu, C. T cell antigen recognition at the cell membrane. Mol. Immunol. 52, 155-164 (2012).

[8] Frank, S. A. Immunology and evolution of infectious disease, (Princeton University Press, Princeton, 2002).

[9] Kersh, G. J., Kersh, E. N., Fremont, D. H. \& Allen, P. M. Highand low-potency ligands with similar affinities for the TCR: the importance of kinetics in TCR signaling. Immunity 9,817 826 (1998).

[10] Evavold, B. D. \& Allen, P. M. Separation of IL-4 production from $\mathrm{Th}$ cell proliferation by an altered $\mathrm{T}$ cell receptor ligand. Science 252, 1308-1310 (1991).

[11] Dittel, B. N., Sant'Angelo, D. B. \& Janeway, C. A. Peptide antagonists inhibit proliferation and the production of IL-4 
and/or IFN-gamma in T helper 1, T helper 2, and T helper 0 clones bearing the same TCR. J. Immunol. 158, 4065-4073 (1997).

[12] Monks, C. R., Freiberg, B. A., Kupfer, H., Sciaky, N. \& Kupfer, A. Three-dimensional segregation of supramolecular activation clusters in T cells. Nature 395, 82-86 (1998).

[13] Rudolph, M. G. \& Wilson, I. A. The specificity of TCR/pMHC interaction. Curr. Opin. Immunol. 14, 52-65 (2002).

[14] Rudolph, M. G., Stanfield, R. L. \& Wilson, I. A. How TCRs bind MHCs, peptides, and coreceptors. Annu. Rev. Immunol. 24, 419-466 (2006).

[15] Wang, Q., Zhang, P., Hoffman, L., Tripathi, S., Homouz, D., Liu, Y., Waxham, M. N. \& Cheung, M. S. Protein recognition and selection through conformational and mutually induced fit. Proc. Natl. Acad. Sci. USA 110, 20545-20550 (2013).

[16] Smith-Garvin, J. E., Koretzky, G. A. \& Jordan, M. S. T cell activation. Annu. Rev. Immunol. 27, 591-619 (2009).

[17] Liedberg, B., Nylander, C. \& Lunström, I. Surface plasmon resonance for gas detection and biosensing. Sensors and Actuators 4, 299-304 (1983).

[18] Homola, J., Yee, S. S. \& Gauglitz, G. Surface plasmon resonance sensors: review. Sensors Actuators B Chem. 54, 3-15 (1999).

[19] Matsui, K., Boniface, J. J., Steffner, P., Reay, P. A. \& Davis, M. M. Kinetics of T-cell receptor binding to peptide/I-Ek complexes: correlation of the dissociation rate with T-cell responsiveness. Proc. Natl. Acad. Sci. USA 91, 12862-12866 (1994).

[20] Lyons, D. S., Lieberman, S. A., Hampl, J., Boniface, J., Chien, Y. H., Berg, L. J. \& Davis, M. M. A TCR binds to antagonist ligands with lower affinities and faster dissociation rates than to agonists. Immunity 5, 53-61 (1996).

[21] Huppa, J. B., Axmann, M., Mortelmaier, M. A., Lillemeier, B. F., Newell, E. W., Brameshuber, M., Klein, L. O., Schutz, G. J. \& Davis, M. M. TCR-peptide-MHC interactions in situ show accelerated kinetics and increased affinity. Nature 463, 963-967 (2010).

[22] Grakoui, A., Bromely, S. K., Sumen, C., Davis, M. M., Shaw, A. S., Allen, P. M. \& Dustin, M. L. The Immunological Synapse: a molecular machine controlling T cell activation. Science 285, 221-227 (1999).

[23] Stone, J. D., Chervin, A. S. \& Kranz, D. M. T-cell receptor binding affinities and kinetics: impact on T-cell activity and specificity. Immunology 126, 165-176 (2009).

[24] Aleksic, M., Dushek, O., Zhang, H., Shenderov, E., Chen, J. L., Cerundolo, V., Coombs, D. \& van der Merwe, P. A. Dependence of $\mathrm{T}$ cell antigen recognition on $\mathrm{T}$ cell receptorpeptide MHC confinement time. Immunity 32, 163-174 (2010).

[25] Dushek, O., Aleksic, M., Wheeler, R. J., Zhang, H., Cordoba, S. P., Peng, Y.C., Chen, J.L., Cerundolo, V., Dong, T., Coombs, D. \& van der Merwe, P. A. Antigen potency and maximal efficacy reveal a mechanism of efficient T cell activation. Sci. Signal. 4, ra39 (2011).

[26] Hopfield, J. J. Kinetic proofreading: a new mechanism for reducing errors in biosynthetic processes requiring high specificity. Proc. Natl. Acad. Sci. USA 71, 4135-4139 (1974)

[27] Ninio, J. Kinetic amplification of enzyme discrimination. Biochimie 57, 587-595 (1975).

[28] McKeithan, T. W. Kinetic proofreading in T-cell receptor signal transduction. Proc. Natl. Acad. Sci. USA 92, 5042-5046 (1995).

[29] Kersh, E. N., Shaw, A. S. \& Allen, P. M. Fidelity of T cell activation through multistep T cell receptor zeta phosphorylation. Science 281, 572-575 (1998).

[30] George, A. J. T., Stark, J. \& Chan, C. Understanding specificity and sensitivity of T-cell recognition. Trends Immunol. 26, 653-659 (2005)
[31] Feinerman, O., Germain, R. N. \& Altan-Bonnet, G. Quantitative challenges in understanding ligand discrimination by alphabeta T cells. Mol. Immunol. 45, 619-631 (2008).

[32] Coward, J., Germain, R. N. \& Altan-Bonnet, G. Perspectives for computer modeling in the study of T cell activation. Cold Spring Harb. Perspect. Biol. 2, a005538 (2010).

[33] Coombs, D., Dushek, O. \& Merwe, P. A. V. D. A Review of Mathematical Models for T Cell Receptor Triggering and Antigen Discrimination. in Math. Model. Immune Cell Biol. (Molina-París, C. \& Lythe, G. eds.), pp. 25-46 (Springer New York, New York, 2011).

[34] Irvine, D. J., Purbhoo, M. A., Krogsgaard, M. \& Davis, M. M. Direct observation of ligand recognition by $\mathrm{T}$ cells. Nature 419, 845-849 (2002).

[35] Huang, J., Brameshuber, M., Zeng, X., Xie, J., Li, Q. J., Chien, Y. H., Valitutti, S. \& Davis, M. M. A single peptide-major histocompatibility complex ligand triggers digital cytokine secretion in CD4(+) T cells. Immunity 39, 846-857 (2013).

[36] Stefanová, I., Hemmer, B., Vergelli, M., Martin, R., Biddison, W. E. \& Germain, R. N. TCR ligand discrimination is enforced by competing ERK positive and SHP-1 negative feedback pathways. Nat. Immunol. 4, 248-254 (2003).

[37] Altan-Bonnet, G. \& Germain, R. N. Modeling T cell antigen discrimination based on feedback control of digital ERK responses. PLoS Biol. 3, e356 (2005).

[38] Feinerman, O., Veiga, J., Dorfman, J. R., Germain, R. N. \& Altan-Bonnet, G. Variability and robustness in T cell activation from regulated heterogeneity in protein levels. Science 321, 1081-1084 (2008).

[39] Dushek, O. \& Coombs, D. Analysis of serial engagement and peptide-MHC transport in $\mathrm{T}$ cell receptor microclusters. Biophys. J. 94, 3447-3460 (2008).

[40] Dushek, O. \& van der Merwe, P. A. An induced rebinding model of antigen discrimination. Trends Immunol. 35, 153158 (2014).

[41] Krummel, M. F., Sjaastad, M. D., Wülfing, C. \& Davis, M. M. Differential Clustering of CD4 and CD3zeta During T Cell Recognition. Science 289, 1349-1352 (2000).

[42] Campi, G., Varma, R. \& Dustin, M. L. Actin and agonist MHC-peptide complex-dependent T cell receptor microclusters as scaffolds for signaling. J. Exp. Med. 202, 1031-1036 (2005).

[43] Varma, R., Campi, G., Yokosuka, T., Saito, T. \& Dustin, M. L. $\mathrm{T}$ cell receptor-proximal signals are sustained in peripheral microclusters and terminated in the central supramolecular activation cluster. Immunity 25, 117-127 (2006).

[44] Manz, B. N., Jackson, B. L., Petit, R. S., Dustin, M. L. \& Groves, J. T-cell triggering thresholds are modulated by the number of antigen within individual T-cell receptor clusters. Proc. Natl. Acad. Sci. USA 108, 9089-9094 (2011).

[45] Valitutti, S., Müller, S., Cella, M., Padovan, E. \& Lanzavecchia, A. Serial triggering of many T-cell receptors by a few peptideMHC complexes. Nature 375, 148-151 (1995).

[46] Valitutti, S., Coombs, D. \& Dupré, L. The space and time frames of $\mathrm{T}$ cell activation at the immunological synapse. FEBS Lett. 584, 4851-4857 (2010).

[47] Govern, C. C., Paczosa, M. K., Chakraborty, A. K. \& Huseby, E. S. Fast on-rates allow short dwell time ligands to activate T cells. Proc. Natl. Acad. Sci. USA 107, 8724-8729 (2010).

[48] François, P., Voisinne, G., Siggia, E. D., Altan-Bonnet, G. \& Vergassola, M. Phenotypic model for early T-cell activation displaying sensitivity, specificity, and antagonism. Proc. Natl. Acad. Sci. USA 110, E888-E897 (2013).

[49] Lipniacki, T., Hat, B., Faeder, J. R. \& Hlavacek, W. S. Stochastic effects and bistability in $\mathrm{T}$ cell receptor signaling. J. Theor. Biol. 254, 110-122 (2008). 
[50] Mempel, T. R., Henrickson, S. E. \& Von Andrian, U. H. T-cell priming by dendritic cells in lymph nodes occurs in three distinct phases. Nature 427, 154-159 (2004).

[51] Yokosuka, T., Sakata-Sogawa, K., Kobayashi, W., Hiroshima, M., Hashimoto-Tane, A., Tokunaga, M., Dustin, M. L. \& Saito, T. Newly generated $\mathrm{T}$ cell receptor microclusters initiate and sustain T cell activation by recruitment of Zap70 and SLP-76. Nat. Immunol. 6, 1253-1262 (2005).

[52] Murugan, A., Huse, D. A. \& Leibler, S. Speed, dissipation, and error in kinetic proofreading. Proc. Natl. Acad. Sci. USA 109, 12034-12039 (2012).

[53] Tu, Y. Quantitative modeling of bacterial chemotaxis: signal amplification and accurate adaptation. Annu. Rev. Biophys. 42, 337-359 (2013).

[54] Shibata, T. \& Ueda, M. Noise generation, amplification and propagation in chemotactic signaling systems of living cells. Biosystems 93, 126-132 (2008).
[55] Namba, T., Nishikawa, M. \& Shibata, T. The relation of signal transduction to the sensitivity and dynamic range of bacterial chemotaxis. Biophys. J. 103, 1390-1399 (2012).

[56] Kobayashi, T. J. Implementation of dynamic bayesian decision making by intracellular kinetics. Phys. Rev. Lett. 104, 228104 (2010).

[57] Kobayashi, T. J. Connection between noise-induced symmetry breaking and an information-decoding function for intracellular networks. Phys. Rev. Lett. 106, 228101 (2011).

[58] Lan, G., Sartori, P., Neumann, S., Sourjik, V. \& Tu, Y. The energy-speed-accuracy tradeoff in sensory adaptation. Nat. Phys. 8, 422-428 (2012).

[59] Sagawa, T. \& Ueda, M. Second law of thermodynamics with discrete quantum feedback control. Phys. Rev. Lett. 100, 080403 (2008).

[60] Ito, S. \& Sagawa, T. Information thermodynamics on causal networks. Phys. Rev. Lett. 111, 180603 (2013). 